\title{
Evolution of Efimov States in 2n Halo Nuclei: A general study
}

Indranil Mazumdar*ł

TUNL and Duke University, Durham, NC 27708

E-mail: indra@tunl.duke.edu

We present an overview of our three-body model calculations to search for the occurrence of Efimov states in 2-neutron halo nuclei. The formalism utilises finite range, separable, non-local interactions to investigate a three-body system where the two halo neutrons are very weakly coupled to the heavy and compact core. The results of the bound state calculation show that a nonBorromean nucleus, like, ${ }^{20} \mathrm{C}$ is more vulnerable to admit Efimov states for a realistic, finite range two-body n-core interaction than Borromean nuclei, such as, ${ }^{14} \mathrm{Be},{ }^{19} \mathrm{~B}$ and ${ }^{22} \mathrm{C}$. Extending the formalism to scattering sector it has been shown that as the two-body (n-core) interaction increases the bound Efimov states in ${ }^{20} \mathrm{C}$ move above the breakup threshold and show up as resonances. The typical asymmetric profile of such resonances has been interpreted in terms of the Fano mechanism and is considered to be a diagnostic tool for possible experimental observation of Efimov states in such nuclei. The phenomenon, so observed for ${ }^{20} \mathrm{C}$, has also been seen in non-Borromean nuclei, like, ${ }^{36} \mathrm{Mg},{ }^{32} \mathrm{Ne}$ and for a hypothetical case of a $2 \mathrm{n}$ halo nucleus with a very heavy core $(A=100)$. In summary, a very consistent picture has emerged from our model calculations for the occurrence of Efimov states and their evolution in to asymmetric resonances for non-Borromean $2 \mathrm{n}$ halo nuclei.

The 7th International Workshop on Chiral Dynamics, August 6 -10, 2012

Jefferson Lab, Newport News, Virginia, USA

\footnotetext{
* Speaker.

${ }^{\dagger}$ The author gratefully acknowledges a long-standing collaboration with V Arora, VS Bhasin, and ARP Rau.
} 


\section{Introduction}

The Efimov effect is a purely quantum mechanical phenomenon where a three-body system has an infinite number of bound states if the two-body interactions marginally (just) bind the binary sub-systems. Efimov showed that if three non-relativistic, identical bosons interact via short-range, attractive two-body interaction $-\lambda v(r)$, characterised by a range parameter $r_{o}$, then as the coupling constant $\lambda$ increases to the value $\lambda_{c}$ that can support the first zero-energy two-particle bound state, the number of bound states of the three-particle system increases without limit as $\mathrm{N} \approx \pi^{-1} \log \left(|a| / r_{o}\right.$ ), where $a$ is the two-particle scattering length that tends to infinity as $\lambda \rightarrow \lambda_{c}$ [1]. The infinite number of three-body bound states disappear rapidly as the coupling constant becomes more attractive. According to Efimov the physical cause of the effect is in the emergence of an effective, attractive, long range force in the three-body system. Efimov studied the three-body system in hyperspherical coordinates and investigated the long-ranged behaviour of the wave function. He concluded that the effective three-body interaction develops a long-range $1 / \mathrm{R}^{2}$ tail, that is responsible for the infinite number of bound states in the Efimov limit. A detailed and rigorous proof of the phenomenon as well as a justification for the disappearance of the three-body bound states with increasing potential strength was published by Amado and Noble based on three-body Faddeev equations almost immediately after Efimov [2]. They studied the eigenvalue spectrum of the Faddeev kernel in a certain singular limit and demonstrated that the kernel of the Faddeev equations becomes non-compact in the Efimov case. While Amado and Noble considered the divergence of the Faddeev kernels in the momentum space, Fonseca et al. showed that the Efimov effect can be understood as a long-range effect in the physical coordinate space [3]. Inspite of the impressive body of theoretical work the experimental observation of Efimov effect has turned out to be highly formidable.

The first experimental observation of Efimov states in an ultracold gas of Caesium atoms has been reported by Kraemer et al. [4]. More recently, Zaccanti et al. have reported observation of an Efimov spectrum in an ultracold system of ${ }^{39} \mathrm{~K}$ atoms [5].

The experimental observation of Efimov states in atomic systems has thrown the challenge to nuclear physicists to search for the effect in atomic nuclei. The remarkable advancement in Radioactive Ion Beam (RIB) physics and the discovery of two-neutron halo nuclei have rejuvenated the search of Efimov states in atomic nuclei. It is now well accepted that the loosely-bound twoneutron halo nuclei are the ideal candidates to search for Efimov or Efimov like states.

Using a three-body model of the two-neutron halo nucleus we have carried out a systematic search for Efimov states in a host of two-neutron halo nuclei. We have also investigated the evolution of the Efimov state in to resonances as the two-body interaction is increased. The results of our calculations are summarised in this report.

\section{Formalism}

We consider a simple three-body model of the 2-neutron halo nucleus comprised of a compact core and two loosely bound valence neutrons. The calculations are carried out in momentum space employing separable potentials for the binary n-n and n-core interactions. Considering s-wave interactions for both n-n and n-core binary sub-systems the three-body wave function is written as

$$
\psi\left(\vec{p}_{12}, \vec{p}_{13} ; E\right)=D^{-1}\left(\vec{p}_{12}, \vec{p}_{3} ; E\right)\left[g\left(\vec{p}_{12}\right) F\left(\vec{p}_{3}\right)+f\left(\vec{p}_{23}\right) G\left(\vec{p}_{1}\right)+f\left(\vec{p}_{31}\right) G\left(\vec{p}_{2}\right)\right]
$$


where

$$
D\left(\vec{p}_{12}, \vec{p}_{3} ; E\right) \equiv \vec{p}_{12}^{2} / 2 \mu_{12}+\vec{p}_{3}^{2} / 2 \mu_{12,3}-E
$$

represents the three-body energy term with total energy $E$ in the three-body c.m system. Here $\vec{p}_{12}$ is the relative momentum of light particles labeled as 1 and $2, \mu_{12}$ is the reduced mass, $\vec{p}_{3}$ is the relative momentum of the core ( labeled as 3 ) with respect to the c.m of the other two and $\mu_{12-3}$ is the corresponding reduced mass. The two-body functions $\mathrm{g}\left(\mathrm{p}_{i j}\right)$ and $\mathrm{f}\left(\mathrm{p}_{i j}\right)$ refer to the s-state separable potentials between the pairs. The details of these potentials are given in [6]. The spectator functions $F(p)$ and $G(p)$ describe respectively the dynamics of the core and of the light halo particles and satisfy the homogeneous coupled integral equations

$$
\begin{gathered}
{\left[\Lambda_{n}^{-1}-h_{n}(p)\right] F(\vec{p})=2 \int d \vec{q} K_{1}(\vec{p}, \vec{q}) G(\vec{q})} \\
{\left[\Lambda_{c}^{-1}-h_{c}(p)\right] G(\vec{p})=\int d \vec{q} K_{2}(\vec{p}, \vec{q}) F(\vec{q})+\int d \vec{q} K_{3}(\vec{p}, \vec{q}) G(\vec{q})}
\end{gathered}
$$

where the detailed expressions for the kernels $\mathrm{K}_{1}, \mathrm{~K}_{2}$ and $\mathrm{K}_{3}$ are worked out in [6]. For the purpose of studying the sensitive computational details as warranted in the Efimov effect, these equations are suitably transformed involving only the dimensionless quantities. The resulting equations are then numerically computed as an eigenvalue problem following the procedure described in detail in $[7,8]$ to determine the three body ground state energy as well as the energy of the Efimov states.

\section{3. ${ }^{14} \mathrm{Be},{ }^{19} \mathrm{~B}$ and ${ }^{22} \mathrm{C}$}

The first 2-n halo nucleus studied using this formalism was ${ }^{14} \mathrm{Be}$ considered to be a three-body system of a ${ }^{12} \mathrm{Be}$ core and two loosely bound valence neutrons [7]. Keeping the n-core range parameter fixed the strength parameter was varied corresponding to $\mathrm{n}-{ }^{12} \mathrm{Be}$ virtual states from $50 \mathrm{keV}$ to $0.01 \mathrm{keV}$. At $50 \mathrm{keV}$ virtual state, the three-body system is found to have binding energy close to the experimental value, but no excited state is predicted. As the virtual state energy of $\mathrm{n}^{12} \mathrm{Be}$ is decreased, we not only get the ground state energy, but also the excited state energy for the ${ }^{14} \mathrm{Be}$ system. In fact the first excited state appears for $n-{ }^{12} \mathrm{Be}$ virtual state of about $4 \mathrm{keV}$ followed by the emergence of the second excited state at $\mathrm{n}$-core virtual state of $2 \mathrm{keV}$.

This methodology was followed to search for Efimov states in ${ }^{19} \mathrm{~B},{ }^{22} \mathrm{C}$ and ${ }^{20} \mathrm{C}$ [8]. It was shown by numerical analysis and also from analytical considerations that Borromean-type halo nuclei like ${ }^{19} \mathrm{~B}$ and ${ }^{22} \mathrm{C}$, where $\mathrm{n}-\mathrm{n}$ and $\mathrm{n}$-core are both unbound, are much less vulnerable to respond to the existence of the Efimov effect. On the contrary, those nuclei, like ${ }^{20} \mathrm{C}$ in which the halo neutron is supposed to be in the intruder low lying bound state with the core, appear to be promising candidates to search for the occurrence of Efimov states at energies below the $\mathrm{n}$-(n-core) breakup threshold. 


\section{The case of non-Borromean ${ }^{20} \mathrm{C}$}

The nucleus ${ }^{20} \mathrm{C}$ has been studied by scanning a wide region of the $\mathrm{n}$-core binding energy from 60 to $500 \mathrm{keV}$. It has been noticed that as the two-body binding energy reaches around $140 \mathrm{keV}$, the second Efimov state has its energy less than that of the two-body leading to an unstable state. Similarly, the first Efimov state also becomes unstable for the two-body binding energy around $240 \mathrm{keV}$. This is in conformity with what was originally predicted by Amado and Noble about the movement of Efimov states into the unphysical sheet associated with the two-body unitarity cut on increasing the strength of the binary interaction. This particular behaviour was investigated by extending the study in the scattering sector. We studied the elastic scattering amplitude for $n$ -

${ }^{19} \mathrm{C}$ system as a function of incident neutron energy by computing the integral equations for the amplitude at energies below the three-body break up threshold [9].

For studying the scattering process of $\mathrm{n}$ by a two body bound ( $\mathrm{n}$-core) system, the spectator function $\mathrm{G}(\mathrm{p})$ describing the behavior of light $\mathrm{n}$ in the presence of the other two is subjected to the boundary condition

$$
G(\vec{p})=(2 \pi)^{3} \delta(\vec{p}-\vec{k})+\frac{4 \pi a_{k}(\vec{p})}{p^{2}-k^{2}-\imath \varepsilon}
$$

The scattering amplitude is normalized such that for s-wave scattering

$$
a_{k}(\vec{p})_{|\vec{p}|=|\vec{k}|} \equiv f_{k}=\frac{e^{l \delta} \sin \delta}{k}
$$

It was found that for binding energies greater than or equal to $250 \mathrm{keV}$ for the $\mathrm{n}-{ }^{18} \mathrm{C}$ system the disappearance of the first Efimov state gives rise to a resonance at the neutron energy of $1.6 \mathrm{keV}$ with a full width of around $0.25 \mathrm{keV}$. The same trend was also observed for binding energies of 200 and $350 \mathrm{keV}$ with the resonances appearing at the same position with similar widths of around $0.25 \mathrm{keV}$. The second excited state was also found to disappear above the $\mathrm{n}+{ }^{18} \mathrm{C}$ threshold of about $140 \mathrm{keV}$ with the appearance of a resonance showing the generality of this behaviour.

\section{The Fano mechanism and evolution of Efimov states}

A very intriguing feature of the resonances described in the previous section are their asymmetric profiles. This is unlike the symmetric Breit-Wigner or Lorentzian shapes encountered more often in nuclear physics. We have interpreted the asymmetric shapes of the resonances as Fano resonances widely observed and studied in atomic and molecular systems [10]. The Fano resonances originate due to the presence of two alternative pathways to the final state. One directly into the continuum and the other through the embedded discrete state, interfere both constructively and destructively to give the asymmetric resonance. In ${ }^{20} \mathrm{C}$, the very weak binding and large spatial spread of the Efimov states lead to a strong overlap with the continuum states leading to comparable amplitudes of the two pathways and the very asymmetric profile. We have fitted the resonances by Fano profiles and have extracted the best fit Fano indices for the resonances [11]. The fits to the resonances at 250 and $150 \mathrm{keV} \mathrm{n}-{ }^{18} \mathrm{C}$ binding energies yield the same fano index $(q)$, displaying their origin as members of the same family of Efimov states. 


\section{6. ${ }^{36} \mathrm{Mg}$ and ${ }^{32} \mathrm{Ne}$}

The results presented in the previous section provided ample motivation to generalise the findings. In a very recent work [12] we have enlarged the scope of our ${ }^{20} \mathrm{C}$ study to establish the evolution of Efimov states into resonances for a three-body system consisting of one heavy core and two light particles of equal mass. Scanning of nuclear data tables points to the nuclei ${ }^{32} \mathrm{Ne}$ and ${ }^{38} \mathrm{Mg}$ as exhibiting a structure dynamically similar to ${ }^{20} \mathrm{C}$ [13]. Our numerical analysis [12] confirms this, opening up the possibility for experimentally studying Efimov states in these nuclei as well.

For both these nuclei the 2-n separation energies are comparable to that of ${ }^{20} \mathrm{C}(2570$ and $1970 \mathrm{keV}$, respectively) as are the energies of the n-core systems of ${ }^{37} \mathrm{Mg}$ and ${ }^{31} \mathrm{Ne}$ which are, respectively, nominally bound by 250 and $330 \mathrm{keV}$ [13]. We have carried out detailed calculations for both these nuclei assuming compact cores of ${ }^{36} \mathrm{Mg}$ and ${ }^{30} \mathrm{Ne}$ with two valence neutrons forming the halo in ${ }^{38} \mathrm{Mg}$ and ${ }^{32} \mathrm{Ne}$. For ${ }^{38} \mathrm{Mg}$, the second excited state disappears in the continuum around $120 \mathrm{keV}$ n-core energy and the first excited state disappears around $220 \mathrm{keV}$. In ${ }^{32} \mathrm{Ne}$, a very similar trend continues with the first excited state vanishing around $120 \mathrm{keV}$ and the second just beyond $220 \mathrm{keV}$. These bound state calculations are followed by probing the scattering sector as was done in case of ${ }^{20} \mathrm{C}$. The result of the elastic scattering cross sections for $\mathrm{n}$ and the bound $\mathrm{n}$-core systems for ${ }^{38} \mathrm{Mg}$ and ${ }^{32} \mathrm{Ne}$ are shown in the middle and right panels of Fig. 1., respectively, for n-core interaction energies of 250 and $150 \mathrm{keV}$. These are the regions where the first and the second excited states disappear. The figures show the resonance structures with centroids around 1.5-1.6 keV and widths of $0.5-0.6 \mathrm{keV}$.

In addition to ${ }^{36} \mathrm{Mg}$ and ${ }^{32} \mathrm{Ne}$ we have also studied a hypothetical case of a very heavy core of mass 100 with two valence neutrons. In this case, as shown in the left panel of Fig.1. we find an asymmetric resonance structure with the centroid around $1.5 \mathrm{keV}$ and width of approximately 0.5 $\mathrm{keV}$. This behaviour is remarkably similar to that of $\mathrm{n}-\left({ }^{19} \mathrm{C}\right)$ elastic scattering [9]. We have also checked the scattering length of the $n-($ core $+n)$ system for incident energy near zero and found it to be positive and large, thereby, indicating a bound state. These results for a hypothetical nucleus with a very heavy core (mass $=100$ ) and two valence halo neutrons show the same behaviour as that of a realistic 2-n halo nucleus, ${ }^{20} \mathrm{C}$. This helps to reinforce the results obtained in [9] over a large mass range.

\section{Conclusion}

In conclusion, we have generalized the study of the evolution of Efimov states into the scattering regime in nuclear systems where the two halo neutrons are weakly bound to the core. In particular, we have seen that in the field of 2 n-rich halo nuclei, which are candidates for Efimov states, a resonant state can result from the coupling of an Efimov state in the closed channel to the open channel in scattering as the energy of the incident neutron approaches the excited level. The occurrence of such a resonance with an asymmetric profile should be a characteristic feature of 2n-rich halo nuclei. 

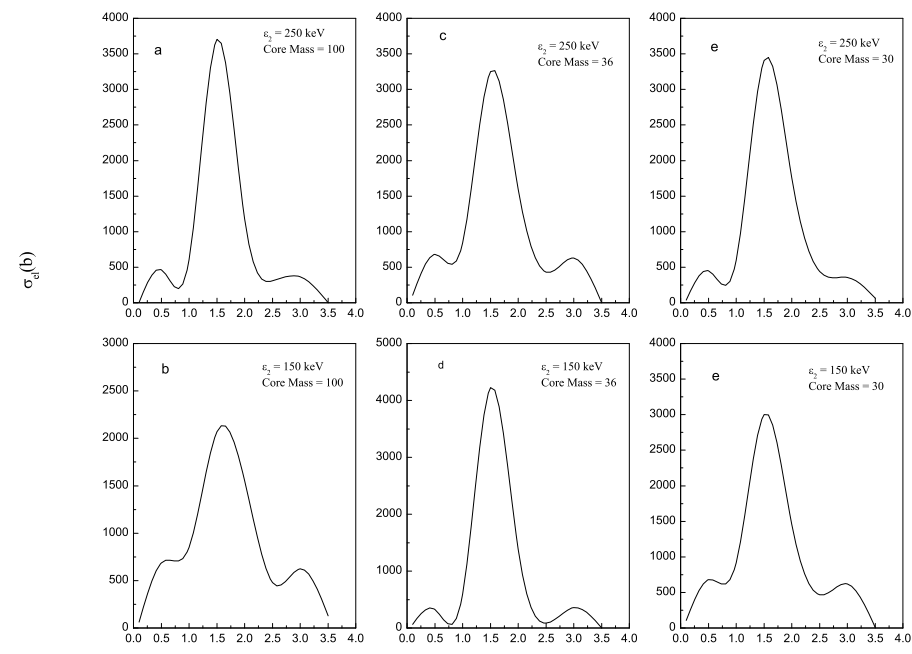

$\mathrm{E}_{\mathrm{i}}(\mathrm{keV})$

Figure 1: Plots of elastic scattering cross sections for nuclei with a very heavy core of 100 (a \& b)), ${ }^{36} \mathrm{Mg}$ core (c \& d), and ${ }^{30} \mathrm{Ne}$ core (e \& f) for two different n-core interaction energies of $250 \mathrm{keV}$ and $150 \mathrm{keV}$.

\section{References}

[1] V. Efimov, Phys. Lett. B33, 563 (1970).

[2] R.D. Amado and J.V. Noble, Phys. Lett. B35, 25 (1971).

[3] A.C. Fonseca et. al. Nucl. Phys. A320, 273 (1979).

[4] T. Kraemer et al., Nature(London) 449, 315(2006).

[5] M. Zaccanti et al., Nature Physics5, 586(2009).

[6] S. Dasgupta, I. Mazumdar, and V.S. Bhasin, Phys. Rev. C50, R550 (1994).

[7] I. Mazumdar, and V.S. Bhasin, Phys. Rev. C 56, R5, (1997).

[8] I. Mazumdar, V. Arora, and V.S. Bhasin, Phys. Rev. C 61, 051303 (2000).

[9] V. Arora, I. Mazumdar, and V. S. Bhasin, Phys. Rev. C 69, 061301 (R) (2004).

[10] U. Fano, Phys. Rev.124,1866 (1961); U. Fano and A.R.P. Rau, Atomic Collisions and spectra (Academic, Orlando, 1986).

[11] I. Mazumdar, A.R.P. Rau, V.S. Bhasin, Phys. Rev. Lett.97, 062503 (2006).

[12] I. Mazumdar, V.S. Bhasin, A.R.P Rau, Phys. Lett. B704 (2011).

[13] G. Audi and A. H. Wapstra, Nucl. Phys. A729, 337 